\title{
Performance Bounds of Opportunistic Scheduling in Wireless Networks
}

\author{
Yanhui Geng, Member, IEEE, Albert Y.S. Lam, Member, IEEE, Victor O.K. Li, Fellow, IEEE \\ Department of Electrical and Electronic Engineering \\ The University of Hong Kong, Hong Kong SAR, China \\ Email: \{yhgeng, ayslam, vli\}@eee.hku.hk
}

\begin{abstract}
In this paper, we study the performance of opportunistic scheduling in wireless networks from the perspective of information and entropy. In opportunistic scheduling, we allocate a limited number of channels to a certain number of nodes so as to maximize the network performance. Due to the inherent uncertainty of the system input represented by random variables with certain probability distributions, even under the optimal scheduling strategy, we may not achieve the best network performance. In our proposed model, we mathematically formulate the relationship between system uncertainty characterized by entropy and network performance, i.e., we give the lower and upper bounds of network performance with given entropy of the uncertain input. Based on this result, we can determine quantitatively the impact of system uncertainty on the performance of of opportunistic scheduling in wireless networks.
\end{abstract}

Index Terms-Optimal entropies, opportunistic scheduling, wireless networks.

\section{INTRODUCTION}

In wireless network opportunistic scheduling, a scheduler has a large number of nodes to serve but there are only a limited number of channels. Therefore, we have to allocate the channels effectively so as to maximize the system performance. Of course, the performance of this system highly depends on uncertain input of the system (e.g. nodes' mobility, channel condition), whose randomness is characterized by its entropy. Therefore, in this paper, we will formulate the relationship between the performance of opportunistic scheduling and the system's uncertain input.

To relate the performance with the entropy of the system, we determine the maximum and minimum entropies for probability distributions of system inputs with constraints. In other words, we figure out the most and least uncertain system inputs with the constraints satisfied. Then we inversely transform the maximum and minimum entropies and obtain the lower and upper bounds of the network performance, respectively, for any given entropy characterizing a class of opportunistic scheduling problems. With these theoretical results, when designing opportunistic scheduling algorithms, we can determine if the potential performance gain worth the additional information collection cost.

A comprehensive overview of opportunistic scheduling algorithms in wireless networks can be found in [1]. As to the study of network performance via an information-theoretical approach, in [2], [3], the authors analyze network performance from the perspective of information quality and information accuracy, but the target application is not specifically on opportunistic scheduling. In [4], maximum entropy is employed to select the hash keys for partitioning the rulebase in the packet classification problem, while a technique based on minimum entropy is developed in [5] to perform carrier frequency recovery in the non-data-aided manner.

The motivation of this work mainly comes from [6] and [7]. In [6], Feder and Merhav studied the relations between entropy and error probability for systems in which only one component (i.e. one channel and one node) is selected and evaluated. The upper and lower bounds of error probability for such systems were derived. The authors of [7] aimed to characterize the performance of three types of online algorithms in terms of entropy. Our work described in this paper is more general and can be used in various applications. Besides, our derived performance bounds are tighter and the computational complexity is greatly reduced than all previous approaches.

The rest of the paper is organized as follows. Section II introduces some preliminary results. In Section III, we first determine the optimum entropies of constrained distribution and then derive the upper and lower bounds of the performance of wireless opportunistic scheduling. Then in Section IV, an opportunistic scheduling protocol is investigated to evaluate the feasibility of our derived results. Finally we conclude in Section V.

\section{PRELIMINARIES}

To facilitate the proofs of later results, we first investigate the properties of function $f_{e}(\cdot)=-x \log x, x \in[0,1]$

Lemma 2.1: $f_{e}(\cdot)$ is strictly concave.

Proof: The second order derivative of $f_{e}(x)$, i.e. $f_{e}^{\prime \prime}(x)=$ $-\frac{1}{x \ln 2}$ is negative $\forall x \in[0,1]$. Thus, $f_{e}(x)$ is strictly concave.

Lemma 2.2: Consider any two points $x_{1}$ and $x_{2}$ in interval $[0,1]$ with $x_{1} \geq x_{2}$, and an arbitrary positive number $\delta$ satisfying $x_{1}+\delta \leq 1$ and $x_{2}-\delta \geq 0$, the inequality

$$
f_{e}\left(x_{1}+\delta\right)+f_{e}\left(x_{2}-\delta\right)<f_{e}\left(x_{1}\right)+f_{e}\left(x_{2}\right)
$$

is always true. 
Proof: Define $\alpha \triangleq \frac{x_{1}-x_{2}}{x_{1}-x_{2}+\delta}$, we can then write,

$$
\begin{aligned}
& x_{1}=\alpha\left(x_{1}+\delta\right)+(1-\alpha) x_{2} \\
& x_{2}=\alpha\left(x_{2}+\delta\right)+(1-\alpha) x_{1}
\end{aligned}
$$

By strict concavity of $f_{e}(\cdot)$, we can write,

$$
\begin{aligned}
& f_{e}\left(x_{1}\right)>\alpha f_{e}\left(x_{1}+\delta\right)+(1-\alpha) f_{e}\left(x_{2}\right) \\
& f_{e}\left(x_{2}\right)>\alpha f_{e}\left(x_{2}+\delta\right)+(1-\alpha) f_{e}\left(x_{1}\right)
\end{aligned}
$$

Then we can get (1) by summing the two inequalities above.

\section{PERFORMANCE BOUNDS}

Notations and definitions used in this paper are listed in

\begin{tabular}{|c|c|}
\hline Symbol & Meaning \\
\hline$X$ & Discrete random variable \\
\hline$P$ & Probability distribution of $X$ \\
\hline$N$ & Number of states of $X$ \\
\hline$M$ & Number of selected states of $X$ \\
\hline$\pi$ & Error probability \\
\hline$H(P)$ & Entropy of distribution $P$ \\
\hline$H_{\max }\left(H_{\min }\right)$ & Maximum (minimum) entropy \\
\hline$P_{\max }\left(P_{\min }\right)$ & Distribution with maximum (minimum) entropy \\
\hline$P(i \rightarrow j)$ & Partial distribution of $X$ from state $i$ to state $j$ \\
\hline$H(P(i \rightarrow j))$ & Partial entropy of $P(i \rightarrow j)$ \\
\hline
\end{tabular}
Table I for reference.

TABLE I

NOTATIONS AND DEFINITIONS

First we will derive the maximum and minimum entropies of constrained uncertain input. With these results obtained, we do an inverse transformation and get the performance bounds for given entropy, which will be used to study the opportunistic scheduling problems in wireless networks.

\section{A. System Model}

Consider a probability distribution of a discrete random variable $X$ with $N$ possible states. Let $p(i)=\operatorname{Pr}\{X=i\}$ for $i \in[1,2, \ldots, N]$. A probability distribution is represented by a vector of $N$ numbers, i.e. $p(i), 1 \leq i \leq N$. Thus,

$$
\sum_{i=1}^{N} p(i)=1
$$

Without loss of generality, we assume, for $i=1,2, \ldots, N-1$

$$
p(i)>p(i+1) \text {. }
$$

Let $\pi$ be the error probability for guessing the value of a discrete random variable, where $0 \leq \pi \leq 1$. The error probability constraint requires the sum of the first $M$ terms to equal $1-\pi$, where $1 \leq M \leq N$, i.e.,

$$
\sum_{i=1}^{M} p(i)=1-\pi
$$

A probability distribution $P(\pi)$ looks like

$$
[\underbrace{p(1), \ldots, p(M)}_{\sum=1-\pi}, \underbrace{p(M+1), \ldots, p(N)}_{\sum=\pi}]
$$

Let $\bar{p}_{1}$ and $\bar{p}_{2}$ be the means of the first $M$ terms and the last $(N-M)$ terms, respectively, i.e., $\bar{p}_{1}=\frac{1-\pi}{M}$ and $\bar{p}_{2}=\frac{\pi}{N-M}$ To have a feasible probability distribution satisfying (5) and (6), we have the following lemma.

Lemma 3.1: A feasible probability distribution must satisfy $\pi \leq 1-\frac{M}{N}$.

Proof: In a feasible region, $\bar{p}_{1} \geq \bar{p}_{2}$ must hold, therefore, $\frac{1-\pi}{M} \geq \frac{\pi}{N-M}$, then we can get Lemma 3.1.

The entropy of a random variable $X$ with a probability mass function $p(x)$ is defined by $H(X)=-\sum_{x} p(x) \log _{2} p(x)$, with $0 \log _{2} 0=0$. Unless stated otherwise, we take the logarithm to the base 2 . The entropy of the distribution given by (7)) is

$$
H(X)=-\sum_{x=1}^{N} p(x) \log p(x) .
$$

For maximization, our aim is to find a probability distribution with the maximum entropy amongst all feasible distributions. Mathematically,

$$
\max _{\substack{p(x), x=1, \ldots N}} H(X)=-\sum_{x=1}^{N} p(x) \log p(x)
$$

subject to

$$
\left\{\begin{array}{l}
\sum_{i=1}^{M} p(i)=1-\pi \\
\bar{p}_{1} \geq \bar{p}_{2} \\
p(1) \geq p(2) \geq \ldots \geq p(M) \geq \ldots \geq p(N) \geq 0 \\
1 \leq M \leq N, 0 \leq \pi \leq 1
\end{array}\right.
$$

Similarly, for minimization, we have (11) subject to (10).

$$
\min _{\substack{p(x), x=1, \ldots N}} H(X)=-\sum_{x=1}^{N} p(x) \log p(x)
$$

\section{B. Lower Bound of the Performance}

We are going to determine the distribution with maximum and minimum entropies. As shown in Lemma 2.1, the entropy function is concave. In other words, we can follow the KuhnTucker conditions to obtain the unique and simple distribution with the maximum entropy. For the minimum entropy, the distribution depends on the values of $N, M$, and $\pi$. They are the extremal points of the corresponding polyhedron.

According to [8], we have the principle of maximum entropy.

Theorem 3.1: The distribution with maximum entropy $P_{\max }(\pi)$ subject to Constraints (10) is given by

$$
[\underbrace{\bar{p}_{1}, \ldots, \bar{p}_{1}}_{M \text { terms }}, \underbrace{\bar{p}_{2}, \ldots, \bar{p}_{2}}_{(N-M) \text { terms }}]
$$


Corollary 3.1: The maximum entropy of the probability distribution subject to Constraints (10) is given by

$$
H_{\text {max }}=(1-\pi) \cdot \log \left(\frac{M}{1-\pi}\right)+\pi \cdot \log \left(\frac{N-M}{\pi}\right)
$$

Proof: By applying (8) to $P_{\max }(\pi)$, we have

$$
\begin{aligned}
H_{\max }= & -\left[M \frac{1-\pi}{M} \cdot \log \left(\frac{1-\pi}{M}\right)\right. \\
& \left.+(N-M) \frac{\pi}{N-M} \cdot \log \left(\frac{\pi}{N-M}\right)\right]
\end{aligned}
$$

and it further gives the result.

Corollary 3.2:

$$
\pi \geq \frac{H-1-\log M}{\log \left(\frac{N}{M}-1\right)}
$$

Proof: As $H \leq H_{\max }$,

$$
\begin{aligned}
H \leq & (1-\pi) \cdot \log \left(\frac{M}{1-\pi}\right)+\pi \cdot \log \left(\frac{N-M}{\pi}\right) \\
= & (1-\pi) \log M-(1-\pi) \log (1-\pi) \\
& +\pi \log (N-M)-\pi \log \pi
\end{aligned}
$$

As $0 \leq-(1-\pi) \log (1-\pi)-\pi \log \pi \leq 1$,

$$
H \leq \log M+\pi \log \frac{N-M}{M}+1
$$

Then we have the result.

\section{Upper Bound of the Performance}

When $M=1$, we have the following lemma:

Lemma 3.2: As stated in [6], when $M=1$, the probability distribution with the minimum entropy is achieved by $P_{\text {min }}(\pi)=[p(1), \ldots, p(N)]$, where,

$$
\begin{aligned}
& p(1)=1-\pi, \quad p(2)=\pi, \\
& p(3)=\cdots=p(N)=0 \text {, } \\
& 0 \leq \pi \leq \frac{1}{2} \\
& p(1)=p(2)=1-\pi, \quad p(3)=2 \pi-1, \\
& p(4)=\cdots=p(N)=0 \text {, } \\
& \frac{1}{2} \leq \pi \leq \frac{2}{3}
\end{aligned}
$$

$p(1)=\cdots=p(N-1)=1-\pi$,

$p(N)=1-(N-1)(1-\pi) \quad \frac{N-2}{N-1} \leq \pi \leq \frac{N-1}{N}$

We are going to determine the distribution with the minimum entropy for $M \geq 2$. We can divide $P$ into two separate segments, $P(1 \rightarrow M)$ and $P(M+1 \rightarrow N)$. $P(1 \rightarrow M)$ takes the first $M$ terms from $P$. Suppose the value of $p(M)=\min (p(i), i \in\{1, \ldots, M\})$ is pre-determined and equals $p^{\prime}$. It is trivial to see that $\frac{1-\pi}{M} \geq p^{\prime} \geq \frac{\pi}{N-M}$.

With $p(M)=p^{\prime}$, we can construct $P_{1 \rightarrow M}$ as follows to minimize $H(P(1 \rightarrow M))$. The principle of constructing minimal entropy distribution is to allocate probabilities to make them as nonrandom as possible. In other words, we try to allocate large probabilities to a few states and to assign other states with probabilities as small as possible. For example, distribution $[0.6,0.4,0,0,0]$ has smaller entropy than distribution $[0.3,0.3,0.2,0.1,0.1]$.

Lemma 3.3: With the smallest (also the last) element of $P(1 \rightarrow M)$ fixed to $p^{\prime}$, the optimal distribution $P_{\min }(1 \rightarrow M)$ which minimizes the entropy of $P(1 \rightarrow M)$ is given by

$$
\left\{\begin{array}{l}
p(1)=(1-\pi)-(M-1) \times p^{\prime}, \\
p(2)=\cdots=p(M)=p^{\prime}
\end{array}\right.
$$

Proof: Suppose there is a $P(1 \rightarrow M)$, whose sum is equal to $(1-\pi)$ and $p(1) \geq \ldots \geq p(M)=p^{\prime}$. Consider $p(M-1)>p^{\prime}$ and let $\beta=p(M-1)-p^{\prime}>0$. By Lemma 2.2 , we can always assign $p(M-1)$ to $p^{\prime}$ and $p(1)$ to $p(1)+\beta$ and the resulting entropy becomes smaller. Similarly, we apply Lemma 2.2 to $p(M-2), \ldots, p(2)$, we get (13) and its entropy is minimum.

$P(M+1 \rightarrow N)$ takes the last $(N-M)$ terms from $P$. Suppose the value of $p(M+1)=\max (p(i), i \in\{M+1, \ldots, N\})$ is pre-determined and equals $p^{\prime \prime}$. It is trivial to see that

$$
\frac{1-\pi}{M} \geq p^{\prime} \geq p^{\prime \prime} \geq \frac{\pi}{N-M}
$$

With the value of $p(M+1)$ fixed to $p^{\prime \prime}$, we can construct $P(M+1 \rightarrow N)$ as before to minimize $H(P(M+1 \rightarrow N))$.

Lemma 3.4: With the largest (also the first) element of $P(M+1 \rightarrow N)$ fixed to $p^{\prime \prime}$, the optimal distribution $P_{\text {min }}(M+1 \rightarrow N)$ which minimizes the entropy of $P(M+$ $1 \rightarrow N)$ is given by

$$
\left.\begin{array}{l}
p(M+1)=\cdots=p\left(M+\left\lfloor\frac{\pi}{p^{\prime \prime}}\right\rfloor\right)=p^{\prime \prime}, \\
p\left(M+\left\lceil\frac{\pi}{p^{\prime \prime}}\right\rceil\right)=\pi \bmod p^{\prime \prime}, \\
p\left(M+\left\lceil\frac{\pi}{p^{\prime \prime}}\right\rceil+1\right)=\cdots=p(N)=0 .
\end{array}\right\}
$$

Proof: It can be easily proved by following the same logic flow as in proving Lemma 3.3. Moreover, this theorem can also be proved by straightforward verification of the Karush-KuhnTucker conditions.

To connect $P(1 \rightarrow M)$ and $P(M+1 \rightarrow N)$ together, we have the following lemma:

Lemma 3.5: $\mathbf{p}_{\text {min }}$ must have $p^{\prime}=p^{\prime \prime}$. Let $\hat{p}=p^{\prime}=p^{\prime \prime}$. $\hat{p} \in\left[\frac{\pi}{N-M}, \frac{1-\pi}{M}\right]$.

Proof: As $p(M) \geq p(M+1)$, inequality $p^{\prime} \geq p^{\prime \prime}$ must hold.

Consider a distribution $P_{1}$ with $p^{\prime}>p^{\prime \prime}$. By Lemma 2.2, we can always find a positive real number $\lambda \in\left(0, p^{\prime}-p^{\prime \prime}\right]$, such that we can produce $P_{1}^{\prime}$, which is identical to $P_{1}$ except $p_{1}^{\prime}(1)=p_{1}(1)+\lambda$ and $p_{1}^{\prime}(M)=p^{\prime}-\lambda$, with lower entropy.

Similarly, consider a distribution $P_{2}$ with $p^{\prime}>p^{\prime \prime}$. Let $p_{2}(k)$ be the last non-zero element in $P_{2}=\left[p^{\prime \prime}, p(M+\right.$ $\left.2), \ldots, p_{2}(k), 0, \ldots, 0\right]$. We can always find a positive real number $\xi \in\left(0, p^{\prime}-p^{\prime \prime}\right]$, such that we can produce $P_{2}^{\prime}$, which is identical to $P_{2}$ except $p_{2}^{\prime}(M+1)=p^{\prime \prime}+\xi$ and $p_{2}^{\prime}(k)=p_{2}(k)-\xi$, with lower entropy.

By combining the effects on $\lambda$ and $\xi$, we can deduce that a distribution with $p^{\prime}=p^{\prime \prime}$ has smaller entropy than another with $p^{\prime} \neq p^{\prime \prime}$. The one with the lowest entropy is $P_{\text {min }}$, and thus, $P_{\text {min }}$ must have $\hat{p}=p^{\prime}=p^{\prime \prime}$. With (14), $\hat{p} \in\left[\frac{\pi}{N-M}, \frac{1-\pi}{M}\right]$. 


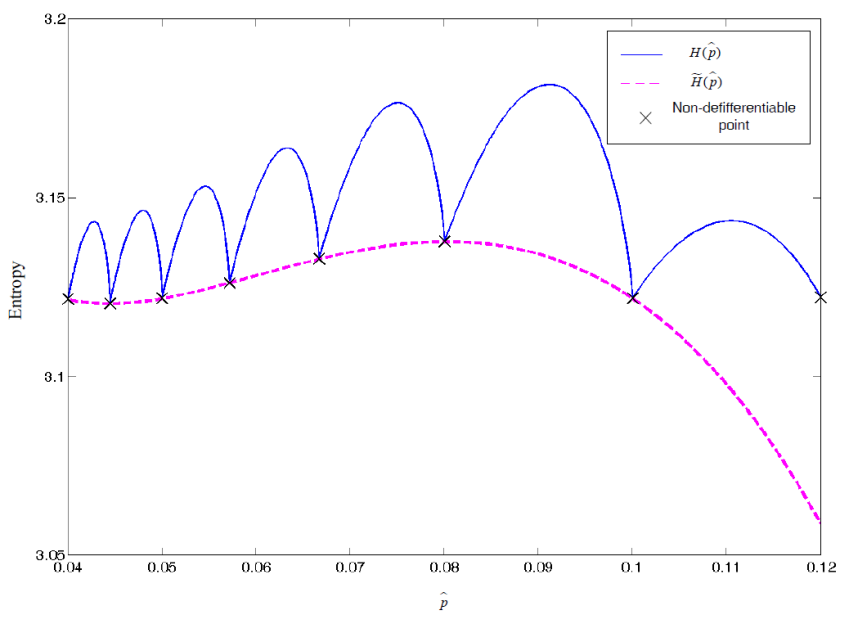

Fig. 1. Plot of $H(\hat{p})$ for $N=15, M=5$, and $\pi=0.4$

Let $y=\left\lceil\frac{N-M-N \pi}{1-\pi}\right\rceil$. From the above results, we have successfully changed the multi-variable optimization of (11) to the single variable optimization as:

$$
\min _{\hat{p} \in\left[\frac{\pi}{N-M}, \frac{1-\pi}{M}\right]} H(\hat{p})=H_{1}(\hat{p})+H_{2}(\hat{p})
$$

subject to (10), with $p(x)$ specified in (13) and (15), where

$$
\begin{aligned}
H_{1}(\hat{p})= & -(M-1) \hat{p} \log \hat{p} \\
& -[(1-\pi)-(M-1) \hat{p}] \log [(1-\pi)-(M-1) \hat{p}]
\end{aligned}
$$

$$
\begin{aligned}
& H_{2}(\hat{p})= \\
& \left\{\begin{array}{l}
-(K) \hat{p} \log \hat{p}, \quad \text { when } \hat{p}=\frac{\pi}{K} \\
-(K-1) \hat{p} \log \hat{p}-[\pi-((K-1) \hat{p})] \log [\pi-((K-1) \hat{p})] \\
\text { when } \frac{\pi}{K}<\hat{p} \leq \frac{\pi}{K-1} \\
-(K-2) \hat{p} \log \hat{p}-[\pi-((K-2) \hat{p})] \log [\pi-((K-2) \hat{p})] \\
\text { when } \frac{\pi}{K-1}<\hat{p} \leq \frac{\pi}{K-2} \\
\vdots \\
-(K-y) \hat{p} \log \hat{p}-[\pi-((K-y) \hat{p})] \log [\pi-((K-y) \hat{p})] \\
\text { when } \frac{\pi}{K-y+1}<\hat{p} \leq \frac{1-\pi}{M}
\end{array}\right.
\end{aligned}
$$

where $K=N-M$.

$H(\hat{p})$ is a continuous function with a piecewise continuous derivative. Its left-end and right-end limits at $\hat{p}$ are $\frac{\pi}{N-M}$ and $\frac{1-\pi}{M}$, respectively. It is composed of a certain number of concave segments and every pair of consecutive concave segments join at a non-differentiable point. We can easily derive from (18) that the function connecting all those nondifferentiable points is,

$$
\tilde{H}(\hat{p})=\pi \log (\hat{p})
$$

$H(\hat{p})$ and $\tilde{H}(\hat{p})$ must meet at $\hat{p}=\frac{\pi}{N-M}$, but they may or may not meet at $\hat{p}=\frac{1-\pi}{M}$. The plots of $H(\hat{p})$ and $\tilde{H}(\hat{p})$ for an example with $N=15, M=5$, and $\pi=0.4$ can be found in Figure 1. The shape of $\tilde{H}(\hat{p})$ depends on the values of $N$,
$M$, and $\pi$. It may be monotonically increasing, monotonically decreasing, monotonically increasing and then decreasing, etc. No matter which shape it is, $\hat{p}^{*}$ constituting the minimum $H(\hat{p})$ must be one of the non-differentiable points or $\frac{1-\pi}{M}$. Let $\hat{P}^{*}$ be the set of $\left\{\frac{\pi}{N-M}, \frac{\pi}{N-M-1}, \ldots, \frac{\pi}{N-M-y+1}, \frac{1-\pi}{M}\right\}$. If $y$ equals $\frac{N-M-N \pi}{1-\pi}$, then $\left|\hat{P}^{*}\right|$ equals $\frac{N-M-N \pi}{1-\pi}$. Otherwise, $\left|\hat{P}^{*}\right|$ equals $\left(\frac{N-M-N \pi}{1-\pi}+1\right)$. We can further transform the original multi-variable optimization with continuous solution set given by (11) to a single variable optimization with discrete set, given by,

$$
\min _{\hat{p} \in \hat{P}^{*}} \quad H(\hat{p})=H_{1}(\hat{p})+H_{2}(\hat{p})
$$

Moreover, $H(\hat{p})$ can now become $H(\hat{p})=$

$$
\left\{\begin{array}{l}
-(N-1) \hat{p} \log \hat{p}-[(1-\pi) \\
\quad-(M-1) \hat{p}] \log [(1-\pi)-(M-1) \hat{p}], \hat{p}=\frac{\pi}{N-M} \\
-(N-2) \hat{p} \log \hat{p}-[(1-\pi) \\
\quad-(M-1) \hat{p}] \log [(1-\pi)-(M-1) \hat{p}], \hat{p}=\frac{\pi}{N-M-1} \\
\vdots \\
-(N-y) \hat{p} \log \hat{p}-[(1-\pi) \\
-(M-1) \hat{p}] \log [(1-\pi)-(M-1) \hat{p}], \hat{p}=\frac{\pi}{N-M-y+1} \\
-(N-y) \hat{p} \log \hat{p}-[\pi \\
-(N-M-y) \hat{p}] \log [\pi-((N-M-y) \hat{p})], \hat{p}=\frac{1-\pi}{M}
\end{array}\right.
$$

Theorem 3.2: The lower bound of the entropy is $\min (A)$, where

$$
A=\left\{\begin{array}{c}
-\frac{(N-1) \pi}{N-M} \log \frac{N-M}{N(N-M)}, \\
-\frac{(N-2) \pi}{N-M-1} \log \frac{N-M}{N(N-M-1)}, \\
\vdots \\
-\frac{(N-y) \pi}{N-M-y+1} \log \frac{N-M}{N(N-M-y+1)}, \\
\frac{(N-y)(1-\pi)}{M} \log M
\end{array}\right\}
$$

Proof: Since $0 \leq(1-\pi)-(M-1) \hat{p} \leq 1$ and $0 \leq$ $\pi-(N-M-y) \hat{p} \leq 1, \hat{p} \in \hat{P}^{*}$, by relaxing (21), we have

$$
H(\hat{p}) \geq \min \left\{\begin{array}{c}
-\frac{(N-1) \pi}{N-M} \log \frac{\pi}{N-M}, \\
-\frac{(N-2) \pi}{N-M-1} \log \frac{\pi}{N-M-1}, \\
\vdots \\
-\frac{(N-y) \pi}{N-M-y+1} \log \frac{\pi}{N-M-y+1}, \\
-\frac{(N-y)(1-\pi)}{M} \log \frac{1-\pi}{M}
\end{array}\right\}
$$

Since $0 \leq \pi \leq 1-\frac{M}{N}$, we can relax the above inequality by replacing $\pi$ in the $\log$ function with $1-\frac{M}{N}$, except in the last row. With $0 \leq-(1-\pi) \log (1-\pi) \leq 1$, we have,

$$
H(\hat{p}) \geq \min \left\{\begin{array}{c}
-\frac{(N-1) \pi}{N-M} \log \frac{N-M}{N(N-M)}, \\
-\frac{(N-2) \pi}{N-M-1} \log \frac{N-M}{N(N-M-1)}, \\
\vdots \\
-\frac{(N-y) \pi}{N-M-y+1} \log \frac{N-M}{N(N-M-y+1)}, \\
\frac{(N-y)(1-\pi)}{M} \log M
\end{array}\right\}
$$




\section{Corollary 3.3:}

$$
\pi \leq \max \left\{\begin{array}{c}
\frac{H \cdot(N-M)}{(N-1) \log \frac{N(N-M)}{N-M}}, \\
\frac{H \cdot(N-M-1)}{(N-2) \log \frac{N(N-M-1)}{N-M},} \\
\vdots \\
\frac{H \cdot(N-M-y+1)}{(N-y) \log \frac{N(N-M-y+1)}{N-M}}, \\
\frac{H \cdot M}{(N-y) \log \frac{1}{M}}+1
\end{array}\right\}
$$

Proof: The result can be directly derived from (23).

Let $\pi_{\min }(H)$ and $\pi_{\max }(H)$ be $\frac{H-1-\log M}{\log \left(\frac{N}{N}-1\right)}$ and the right hand side of inequality (24) respectively. We get the following theorem.

Theorem 3.3: With given entropy of the random variable $X$, for a system with constraints specified in (10), the error probability $\pi$ is bounded by $p i_{\min }(H)$ and $p i_{\max }(H)$ with:

$$
\pi_{\min }(H) \leq \pi \leq \pi_{\max }(H)
$$

Proof: The result is obtained by combining Corollaries 3.2 and 3.3 .

One important benefit of our work is that the computational complexity of finding the optimal $\hat{p}$ is greatly reduced. Originally the solution space is of multiple continuous variables, i.e. (11), and it is simplified to one with a single variable with continuous set $\left[\bar{p}_{2}, \bar{p}_{1}\right]$, i.e. (16). Moreover, recall that the entropy function forms a polyhedron whose minimum points highly depend on the values of $N, M$, and $\pi$. Different combinations of $N, M$, and $\pi$ give different closed-form solutions of minimum entropy. Thus, they are extremely difficult to be found explicitly. Even by using numerical optimization techniques to improve the accuracy and reliability, we need to investigate a large number of discrete points with relatively small step sizes and this will incur heavy computational load. However, according to our analysis, the solution set has shrunk to a limited number of discrete points only and the optimal solution is guaranteed to be one of those points. Furthermore, we can tell from the derived results that going through all the points in our derived set will incur linear computational complexity of $O(N)$.

\section{ILLUSTRATION}

We analyze the lower bound performance of the opportunistic scheduling scheme of a base station-client network. In this network, we have one base station and $N$ nodes. The nodes move around the base station following a mobility model. The connectivity is time slot-based. In other words, in each time slot, we can schedule $M(M \leq N)$ nodes to communicate with the base station. We have a centralized algorithm to control the scheduling. The throughput of each node depends on its channel condition decided by the location of the node.

Consider a particular time slot. Denote by $A$ and $B$ the set of all nodes and the chosen nodes for scheduling, respectively. Then $|A|=N$ and $|B|=M$. In each time slot, each node will enjoy good channel condition with probability $p\left(a_{i}\right)$, and suffer bad channel condition with probability $1-p\left(a_{i}\right)$. Let $p\left(a_{1}\right) \geq p\left(a_{2}\right) \geq \cdots \geq p\left(a_{N}\right)$. Note that $\sum_{i=1}^{N} p\left(a_{i}\right)$ may be larger than 1. But we can perform a transformation as follows to make it fit into our assumption (4).

$$
p\left(b_{i}\right)=\frac{p\left(a_{i}\right)}{\sum_{i=1}^{N} p\left(a_{i}\right)}, 1 \leq i \leq N
$$

According to the best strategy, we should always schedule the $M$ nodes with the highest probabilities of good channel condition to transmit without the consideration of fairness. So the maximum expected system throughput is $U=\sum_{i=1}^{M} p\left(a_{i}\right)$.

This problem fits into our model with $k=M$ and the performance measure is the supplementary of error probability, i.e., $1-\pi$. After transformation and with Corollary 3.2, we can get the following lower bound of the performance.

$$
U \geq \sum_{i=1}^{N} p\left(a_{i}\right) \cdot \frac{H-1-\log (N-M)}{\log \frac{M}{N-M}}
$$

Similarly, we can also get the upper bound of the system performance according to the result of Corollary 3.3. By this example of scheduling, we show that we can give the lower bound and upper bound of the system performance under our proposed framework. We also learn from this illustration that obtaining information about uncertain input or approximating it accurately is very important because of its direct relationship with system performance.

\section{CONCLUSION}

In this paper, we study the performance of opportunistic scheduling in wireless networks from the perspective of information and entropy. First, we derive the maximal and minimal entropies for constrained probability distributions. Based on this result, we do an inverse transformation and get the performance bounds for given entropy. With these foundations established, we analyze the performance of opportunistic scheduling in wireless networks and some meaningful theoretical results are presented. Finally, an opportunistic scheduling application is utilized to illustrate the effectiveness of our work.

\section{REFERENCES}

[1] X. Liu, E. K. P. Chong, and N. B. Shroff, "A framework for opportunistic scheduling in wireless networks," Computer Networks, vol. 41, no. 4, pp. 451-474, 2003

[2] Y. Geng and V. O.K. Li, "A framework to analyze network performance based on information quality," in Proc. IEEE ICC 2010, Cape Town, South Africa, May 2010.

[3] — "Mathematical impact of information accuracy on network performance," in Proc. IEEE ICC 2010, Cape Town, South Africa, May 2010.

[4] L. Choi, H. Kim, S. Kim, and M. H. Kim, "Scalable packet classification through rulebase partitioning using the maximum entropy hashing," IEEE/ACM Trans. Networking, vol. 17, no. 6, pp. 1926-1935, Dec. 2009.

[5] M. Pedzisz and A. Coatanhay, "Minimum entropy approach for carrier frequency recovery," IEEE Trans. Wireless Commun., vol. 5, no. 4, pp. 774-778, Apr. 2006.

[6] M. Feder and N. Merhav, "Relations between entropy and error probability," IEEE Trans. Inf. Theory, vol. 40, no. 1, pp. 259-266, 1994.

[7] G. Pandurangan and E. Upfal, "Entropy-based bounds for online algorithms," ACM Trans. Algorithms, vol. 3, no. 1, p. 7, 2007.

[8] T. M. Cover and J. A. Thomas, Elements of Information Theory, 2nd ed. Wiley-Interscience, June 2006. 